\title{
CURRENT ESTIMATION METHOD AT THE TIME OF A CONTACT-WIRE BREAKAGE DURING STANDSTILL BELOW INSULATED OVERLAP BASED ON CONTACT-WIRE NECKING LENGTH
}

\author{
SHOGO WADA, KAZUHIKO ITO \& TAKAMASA HAYASAKA \\ Power Supply Technology Division, Railway Technical Research Institute, Japan
}

\begin{abstract}
One of the critical points in the DC feeding system is the insulated overlap. In the insulated overlap, the contact-wire breakage accident during standstill sometimes occurred. It is caused by joule heat due to the current flowing between the contact wire and the contact strip, through the arc due to poor contact condition. To understand the phenomenon of this kind of accident and to take countermeasures against it, it is important to evaluate the current that flows through breakage point. However, since the current is affected by the other train load current, it is difficult to estimate it based on the recorded data of the substation. Once the contact wire is softened, its mechanical strength decreases resulting in breakage due to tension force. The authors conducted wire break tests focusing on the arc. As a result, it has been found that the necking of the contact wire at the point of the breakage caused by the arc depends on the arc current and tension of the contact wire. In this study, the relationship between the arc current, the tension of the contact wire, and the necking length of the contact wire was investigated through the tests conducted under the condition where the arc current was set from about 30A to 300A under the tension of $9.8 \mathrm{kN}$ or $19.6 \mathrm{kN}$. Then we proposed a method of estimating the current of the contact wire at the time of its breakage accident during standstill in the insulated overlap section based on the necking length of the contact wire.
\end{abstract}

Keywords: DC electric railways, insulated overlap, contact wire, breaking of wire, arc.

\section{INTRODUCTION}

An insulated overlap is one of the weak points in the DC feeding system. In the insulated overlap, contact-wire breakage accidents during standstill sometimes occurred, which developed into big transport disorders. Therefore, establishment of the effective and low-cost countermeasures against the contact-wire breakage accidents at the insulated overlap are desired by railway operators.

The causes of the contact-wire breakage are joule heat generated by the current flowing between the contact wire and the contact strip, and the arc caused by poor contact between the contact wire and the contact strip. To understand the phenomenon of the accident at this weak point and to take countermeasures against it, it is important to estimate the current flowing through the breakage point. However, intensity of such current is on the level of several hundreds of ampere, which is about the same as that of the train load current. Hence it can't be detected by the measurement device or the failure detection device installed at a substation. Therefore, a method for estimating the current is required. At present, the current is estimated by calculation. On the basis of the train's positions and the train's load current. However, the accuracy of the current estimation by this method is low.

The authors conducted many tests in order to investigate wire breakage properties by an arc. As a result, it is found that there is a correlation between the arc current and the necking length of the contact wire [1]. In this paper, we propose a method of estimating the current flowing between the contact wire and the contact strip through the arc by measurement of the 
necking length of the contact wire based on experimentally obtained relationship between the necking length of the contact wire and the current under some tension conditions.

\section{MECHANISM OF CONTACT-WIRE BREAKAGE BY ARC DURING STANDSTILL}

Fig. 1 shows the mechanism of the contact-wire breakage by the arc during standstill in the case of DC feeding system. Suppose that a contact strip is in contact with the contact wire during standstill and the current is collected. The current flows from the contact wire through the contact strip to the train (a). If the contact between the contact wire and the contact strip is lost, the current continues to flow to the contact strip through an arc (b). When the arc locates at the same place of the contact wire during standstill, the contact wire is locally heated, softened, stretched by tension and necked (c). Finally, the softened and necked portion can't withstand the tension and the contact wire breaks (d).

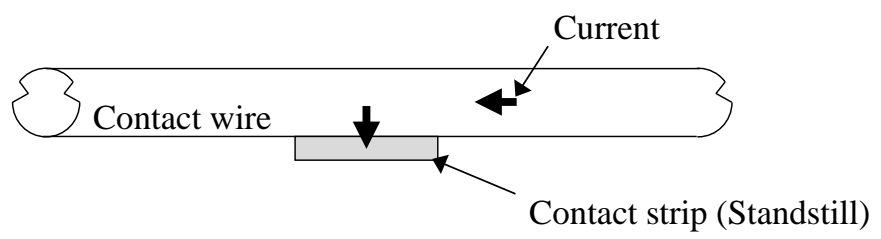

(a) Normal current collection

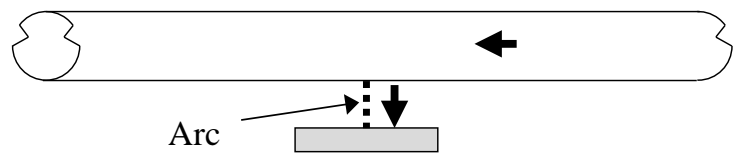

(b) Separation between the contact wire and the contact strip

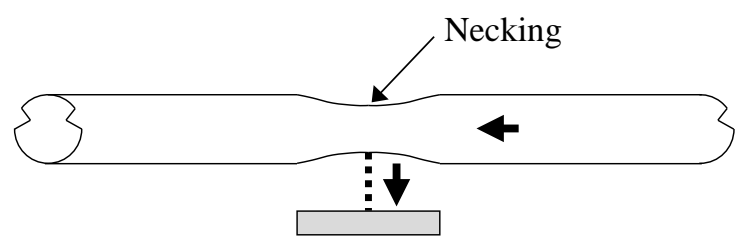

(c) Softening of the contact wire by the arc and necking of the contact wire

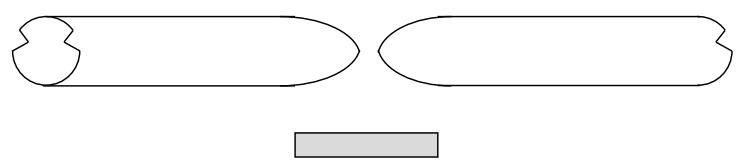

(d) Breakage of the contact wire

Figure 1: Breakage mechanism of the contact wire by the arc. 
The main cause of occurrence of the separation between the contact wire and the contact strip is the stopping of the train at the insulated overlap. The insulated overlap sections two feeding areas by the air gap between the contact lines. It is used for minimizing the blackout area during maintenance or electric accident. As a feature of the insulated overlap, there are several advantages that there is no current interruption at the time of passing through at the pantograph, and it does not become a hard point in collecting the current. Therefore, it is widely adopted as the section suitable for high speed running [2].

The following explains the contact condition between the contact wire and the contact strip when the train passes through the insulated overlap. Fig. 2 shows the outline of the contact state between the contact wire and the contact strip when the train stops at the insulated overlap. Before the train enters the insulated overlap, the train is collecting the current from the line $\mathrm{A}$ in the contact situation. When the train enters the insulated overlap, the B line approaches the contact strip (i), and both the line A and the line B contact with the contact strip at the central portion of the insulated overlap (ii). After the contact strip passes through the central portion of the insulated overlap, the line A gets clear of the contact strip (iii). When the contact strip passes through the insulated overlap, it contacts only with the line B. If the pantograph stops at (i) or (iii), a slight gap occurs between one of the contact wires and the contact strip. Under such case, it is possible that an arc is generated triggered by vibration of train or contact line.

The arc between the contact strip and the contact wire during standstill at the insulated overlap is generated not only due to the current powering the train itself. When another train goes on powering in the same feeding section as shown in Fig. 3, the powering current may flow through the pantograph on the stopped train.

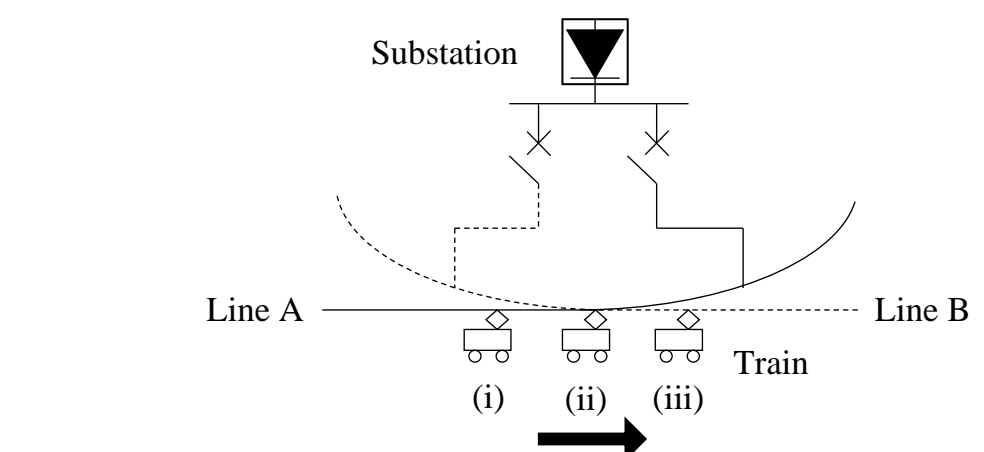

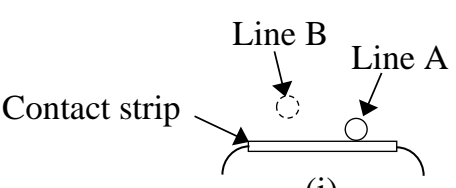

(i)

Direction

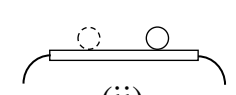

(ii)

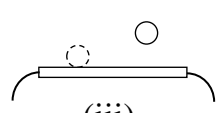

(iii)

(i)(iii) One of the contact wires contact with the contact strip

(ii) Both contact wires contact with the contact strip

Figure 2: Schematic drawing of contact condition between the contact wire and the contact strip at the insulated overlap. 


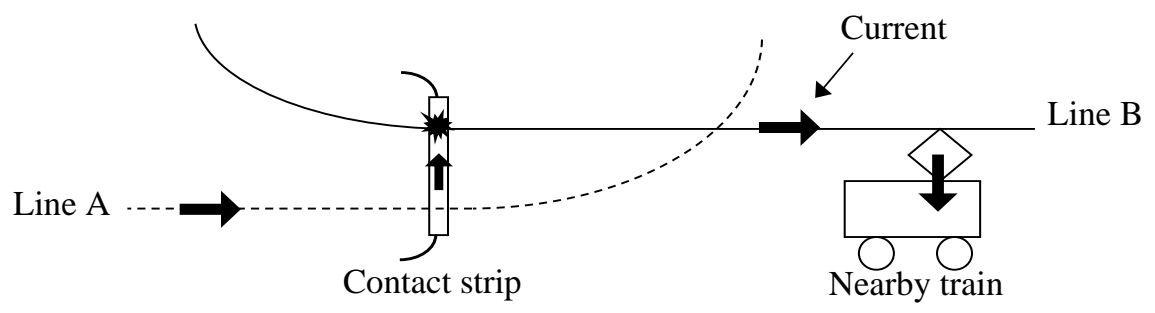

Figure 3: Current flow via the contact strip at the insulated overlap by powering of the nearby train.

\section{EXPERIMENT}

Fig. 4 shows the outline of the experimental apparatus and Fig. 5 shows its circuit diagram. A new wire of GT-SNN110 $\mathrm{mm}^{2}$ which is commonly used in Japan is used as contact wire. The tensions of $9.8 \mathrm{kN}$ and $19.6 \mathrm{kN}$ are pulled by the spring type tensioning device. The contact strip is installed on the actuator which can operate the stage in the vertical direction and is made in contact with the contact wire. Then voltage of $35 \mathrm{~V}$ to $40 \mathrm{~V}$ is applied to the circuit by a DC power supply device (PAT20-400T with two units connected in series, KIKUSUI ELECTRONICS Co.) and a current of 55A to 405A is caused to flow while changing the circuit resistance. Then, the contact strip is lowered and detached from the contact wire by the actuator with moving stage to generate the arc. The tension of the contact wire, the circuit current, the power supply voltage $V_{1}$, and the inter-electrode voltage $V_{2}$ are measured. The descending speed of the contact strip is set at $0.5 \mathrm{~mm} / \mathrm{s}$, and the maximum gap between the contact wire and the contact strip is set at 0.6 to $2 \mathrm{~mm}$. Fig. 6 shows an example of the contact-wire breaking experiment. The contact strip is tilted towards and adjusted so as to make point contact with the sliding surface of the contact wire. The reason is to prevent the arc from moving in the direction of the contact-wire axis when the contact strip is descended. The temperature of the contact wire is also measured. Fig. 7 shows the outline of the temperature measurement. For measurement, a chromel-alumel thermocouple attached to the gripped surface of the contact wire with a heat resistant tape is used. Measurement points are set at $0,20,40$, and $100 \mathrm{~mm}$ in the direction of the axis of the contact wire with the arc occurrence point as the origin.

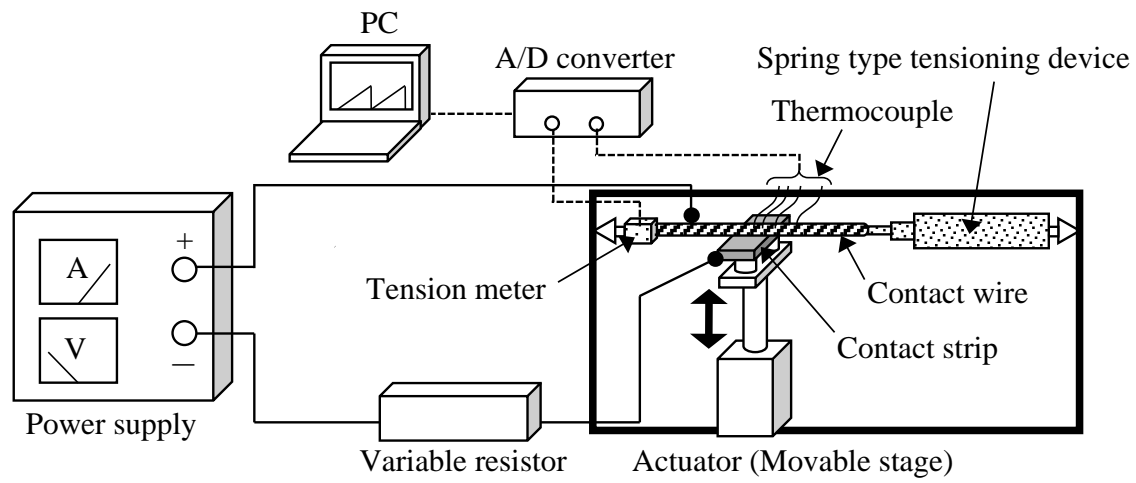

Figure 4: Experimental apparatus. 


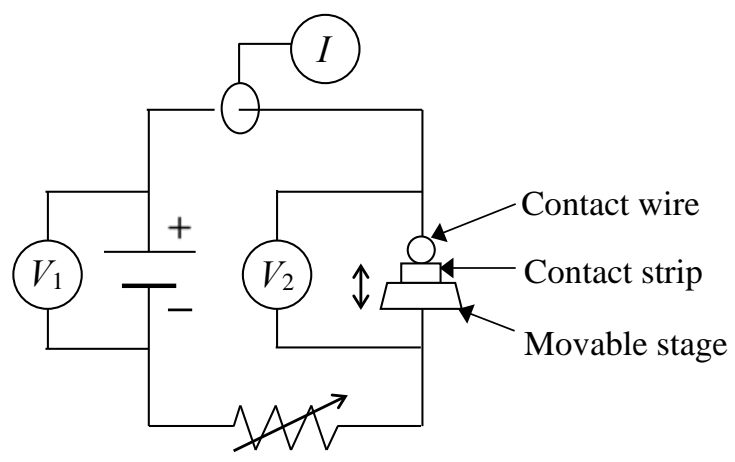

Figure 5: Test circuit and measurement items.

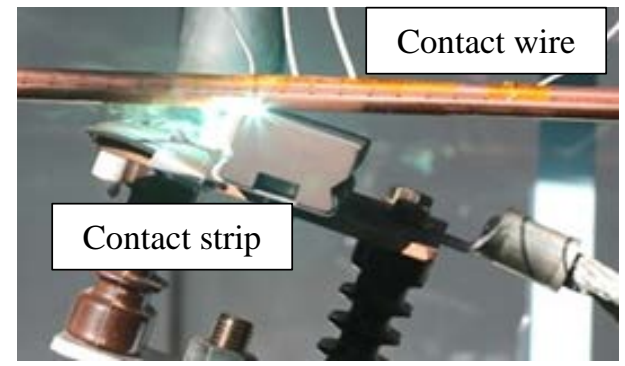

Figure 6: Contact-wire breaking experiment.

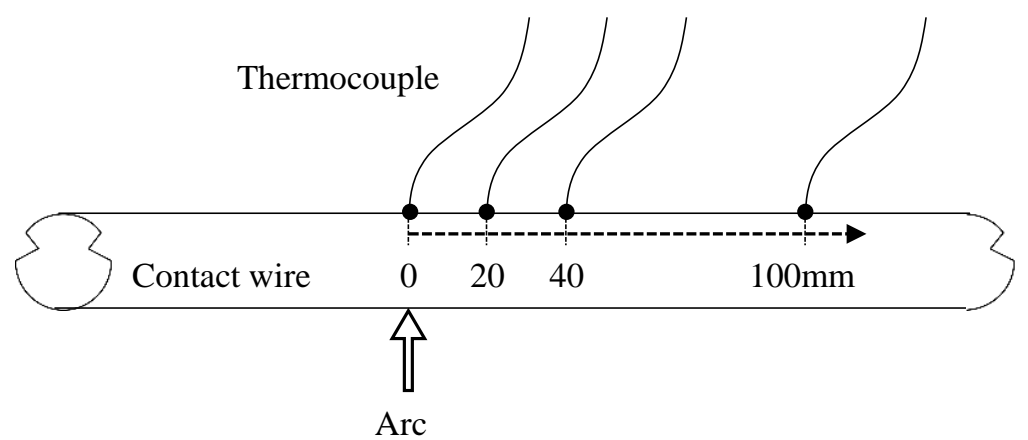

Figure 7: Temperature measurement at the contact-wire surface.

\section{EXPERIMENTAL RESULTS AND DISCUSSION}

\subsection{Measured waveform}

Fig. 8 shows time history of current, voltage and tension force under the condition of tension of $19.8 \mathrm{kN}$, power supply voltage of $37 \mathrm{~V}$ and circuit current of 380A. It starts lowering the 
contact strip at 0.2 seconds. At the same time, the arc occurs between the contact wire and the contact strip, and the inter-electrode voltage $V_{2}$ increases stepwise to $9 \mathrm{~V}$. This is due to the voltage drops occurring at the electrodes at both ends of the arc. At this time, the circuit current decreases to about 300A due to the voltage drop by the arc. After that, the contact wire is broken at 3.1 seconds, the tension decreases, and the arc extinguishes. The arc duration is defined as the time from the moment when $V_{2}$ exceeded $9 \mathrm{~V}$ to the moment when the tension drops, and the arc current and the arc voltage are defined as the average values over the arc duration time. The experimental results are evaluated by these values.

\subsection{Appearance of broken contact wire}

Fig. 9 shows the appearance of the sliding surface of the contact wire broken by a tension of $9.8 \mathrm{kN}$. The tip of the breakage point of the contact wire is softened due to heat arise from the arc, and it is necked stretched by tension. From the appearance of the broken contact wire obtained by the experiment. Shows that the necking area of the contact wire axis becomes shorter under large arc current condition. Furthermore, in the case of the arc current of about $34 \mathrm{~A}$, little damage is observed on the surface of the contact wire, whereas slight damage is observed in case of 121A, and a large crater-like melt marks is observed on the surface in case of 285A. This indicated that as the arc current become higher, the necking area of the contact wire become shorter and the melted marks on the sliding surface of the contact wire become bigger.

\subsection{Necking length of contact wire}

Fig. 10 shows the definition of necking length of the broken contact wire. Necking length is defined as the sum of both breakage contact wire of the distance in the rail direction from the breakage surface to the part with the diameter of the contact wire of $12 \mathrm{~mm}$. And the diameter is measured with a vernier caliper.

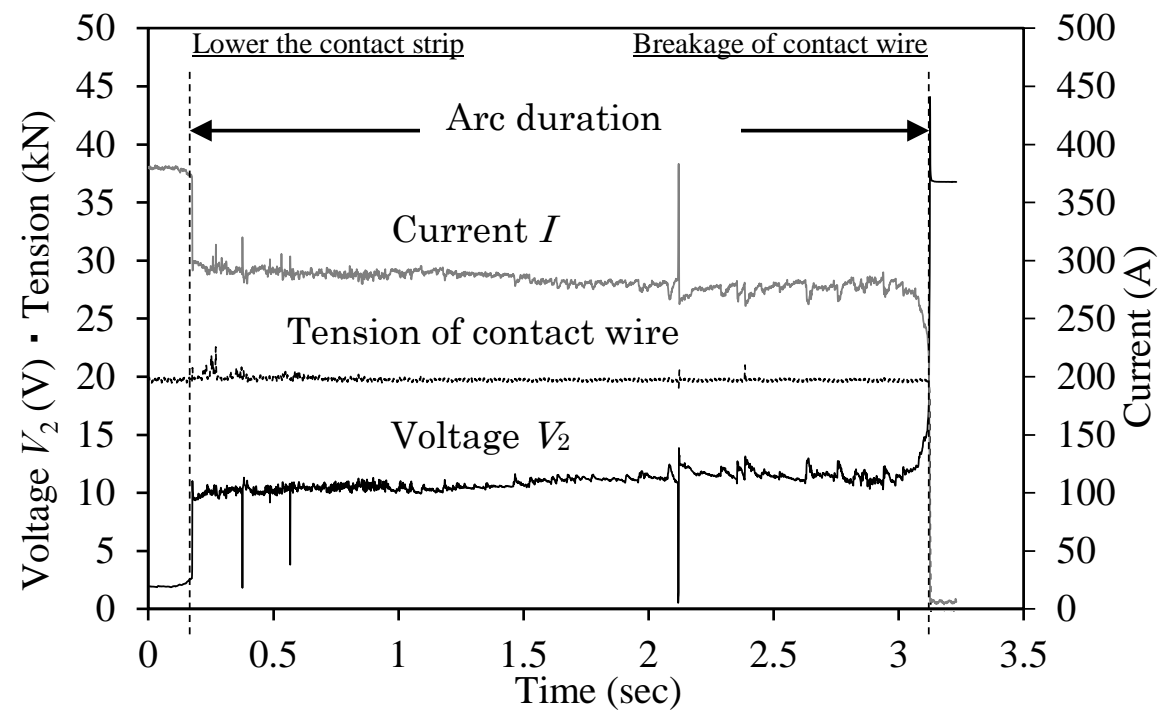

Figure 8: Time history of current, voltage and tension of the contact wire. 


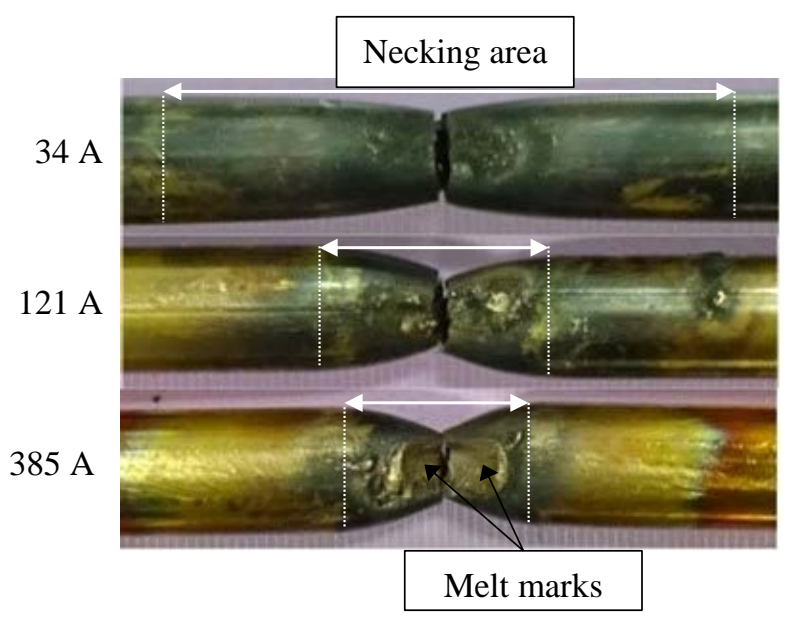

Figure 9: Appearance of the broken contact wires.

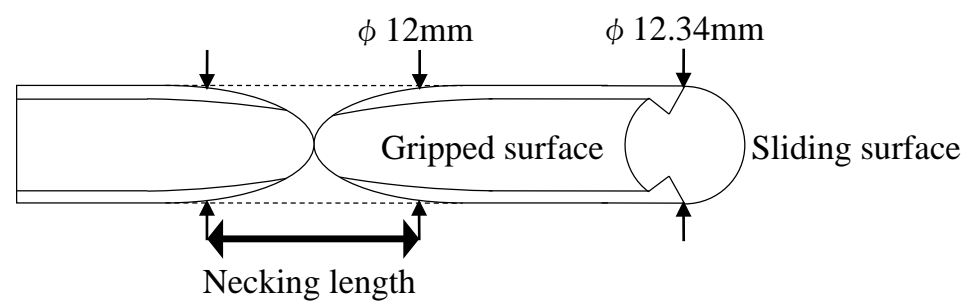

Figure 10: Definition of necking length of the contact wire.

\subsection{Relationship between arc current and arc duration}

Fig. 11 shows the relationship between the arc current and the arc duration obtained in the experiment. In the figure, the approximate curves of experimental results for the respective tensions are shown. The arc duration can be regarded as the time required to break the contact wire. Experimental results show that the arc duration increases as the arc current decreases regardless of tension. In the case of an arc current of about 300A under the tension of $9.8 \mathrm{kN}$, the arc duration is about 5 seconds, whereas in the case of an arc current of 34A under the tension of $9.8 \mathrm{kN}$, the arc duration is 200 seconds. As a result of the experiments under two tension condition, it is found out that as the tension become lower, the arc duration become longer. Furthermore, it is found out that the influence of the tension on the arc duration is small in the case of arc current of about 300A, but that is large in the case of small current about 30A.

\subsection{Relationship between arc current and necking length}

Fig. 12 shows the relationship between the arc current and the necking length of the broken contact wire obtained by the experiment. In the figure, the approximate curve of the 
experimental results for each tension is shown. Although there is dispersion in the experimental results, it is found out that the necking length of the broken contact wire tends to become longer as the arc current becomes smaller. In particular, in the case of low arc current of $100 \mathrm{~A}$ or less, the necking length of the broken contact wire becomes extremely long. According to the experimental results of $9.8 \mathrm{kN}$, the necking length is $17.4 \mathrm{~mm}$ to $23 \mathrm{~mm}$, when the arc current is $313 \mathrm{~A}$ to $121 \mathrm{~A}$. However, it is $46.6 \mathrm{~mm}$ at current of $34 \mathrm{~A}$, which is extremely long. In addition, it is found out that the tension of the contact wire affects the necking length. Fig. 12 indicates that the necking length of the contact wire tends to be longer with the smaller tension. In the experiment at $280 \mathrm{~A}$, the necking length of $9.8 \mathrm{kN}$ is about $4 \mathrm{~mm}$ longer than that of $19.6 \mathrm{kN}$.

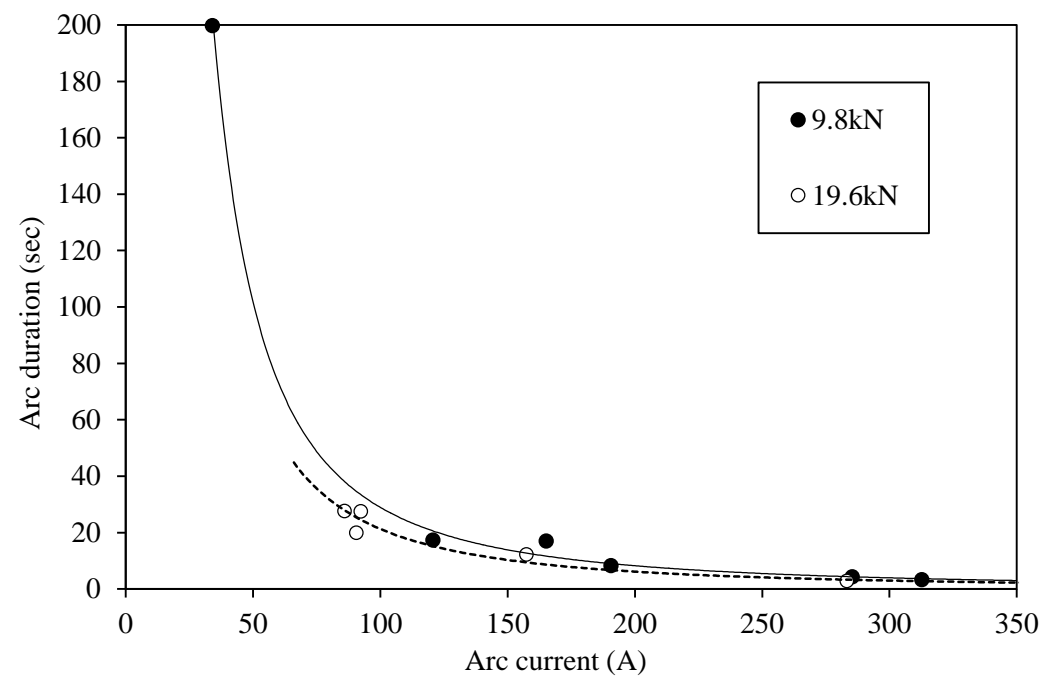

Figure 11: Relationship between arc current and arc duration.

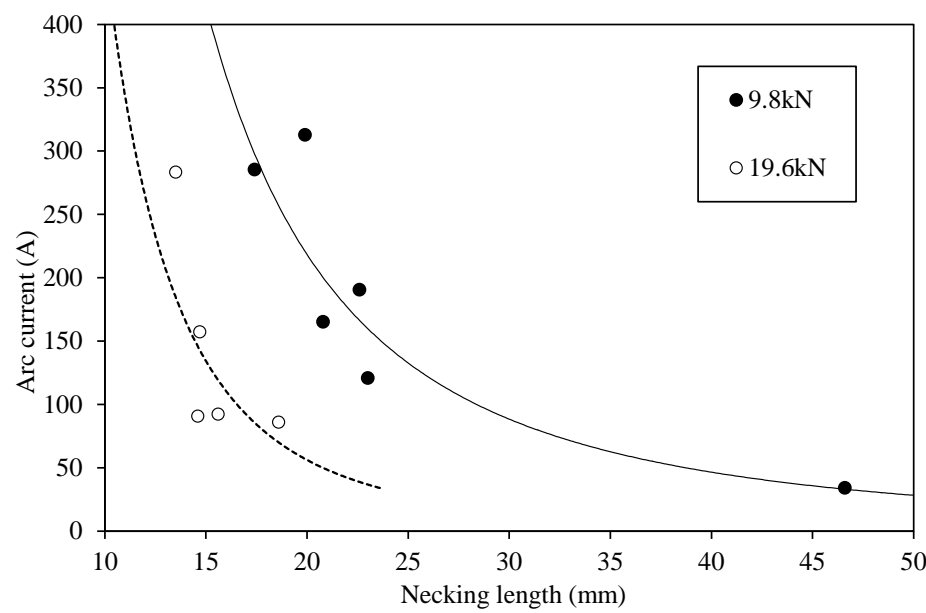

Figure 12: Relationship between necking length of the broken contact wire and arc current. 


\subsection{Temperature of contact wire}

Fig. 13 shows the temperature of the contact wire at each measurement point at the moment of breakage in the case of a tension of $9.8 \mathrm{kN}$. Although the temperature measurement points are only on one side, as easy to understand, the temperature distribution can be assumed to be symmetrical. Therefore, the temperature on both sides of the contact wire with the arc point as the origin is shown in the figure. Furthermore, the softening temperature of the contact wire estimated from the experimental value is shown in the figure. In both results, it is broken when the temperature at the arc point of the gripped surface of the contact wire becomes near $500^{\circ} \mathrm{C}$. However, the temperature at the point $100 \mathrm{~mm}$ away from the arc point in the case of current with 34A is twice as high as that in case of current with 165A. And the former exceeds $200^{\circ} \mathrm{C}$. From this result, it is found that in the case of the current of 34A with 200sec, the temperature of the contact wire increase in a wider range compared to that of $165 \mathrm{~A}$ with $17 \mathrm{sec}$. It can be considered that the necking length is influenced by the total energy of heat input.

From these results, necking length is considered to be closely related to the area of softening by the arc. Fig. 14 shows the temperature distribution of the contact wire when the arc occurs. As the arc current is smaller, the energy for heating the contact wire is likewise smaller. Therefore it takes time to break the contact wire and the arc duration becomes long. In this case, since the total energy input to the contact wire is large, the area where the temperature is raised in the direction of the axis of the contact wire becomes large. As a result, the area softened become wide and is stretched by tension, hence it is considered that the necking length becomes large. The influence of the difference in tension on the necking length can be considered as well. The destruction load of the contact wire is affected by tension and temperature. For example, when the tension is low, the destruction load is reached at a higher temperature than when the tension is high [4]. From this, if the same arc

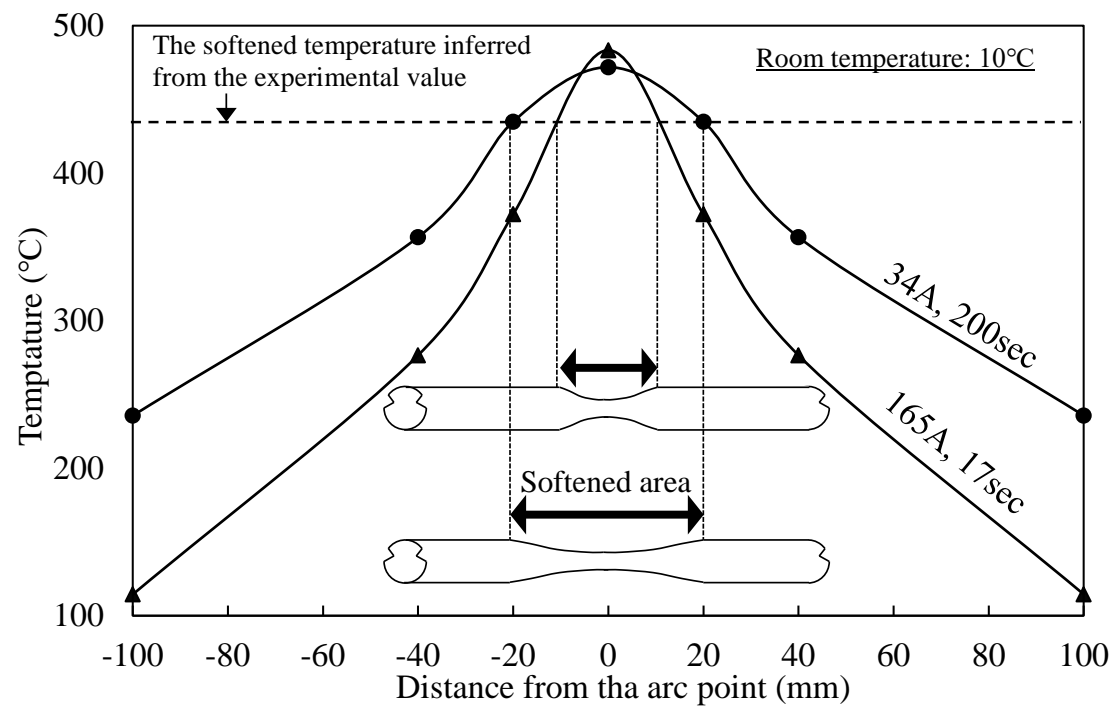

Figure 13: Temperature of the contact wire at each measurement point. 


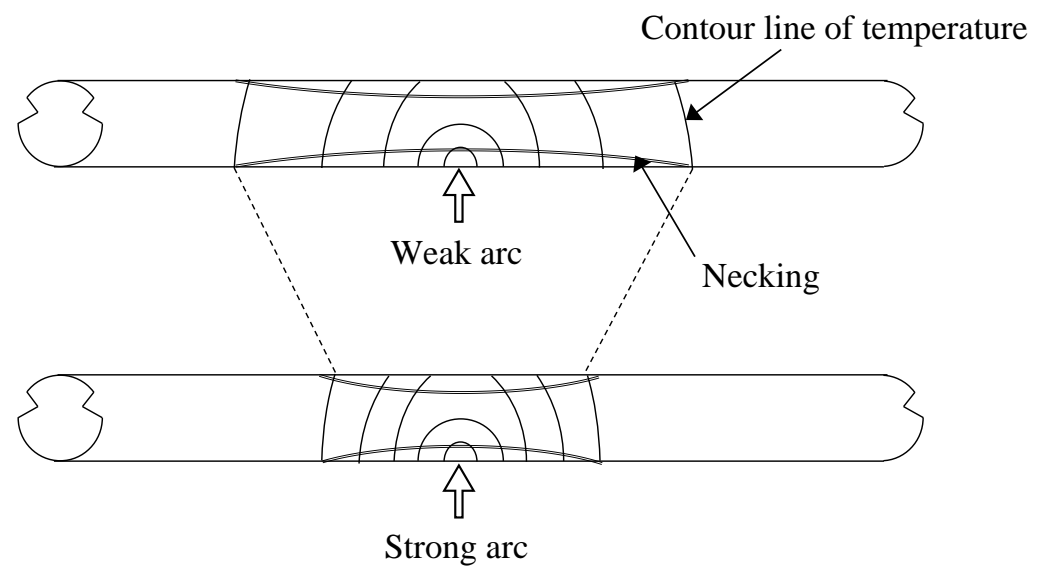

Figure 14: Temperature distribution of the contact wire by the strength of arc.

current is flowed, it takes more time for the contact wire of the smaller tension to break, and the total energy input to the contact wire become large. Therefore, the area where the temperature is raised in the direction of the axis of the contact wire becomes larger. As a result, the area softened become wide and is stretched by tension, hence it is considered that the necking length becomes large.

\section{PROPOSAL OF ESTIMATING THE CURRENT}

Based on these results, we propose an estimation method of the current flowing at breakage point. When investigating the contact-wire breakage accident, the first step is confirmed the melted marks of the surface of the contact wire at the breakage point. If melted marks is not observed on the surface, it is estimated that a small current at breakage of about 100A flowed. If there is a large crater-like melt marks, it is estimated that a large current of about several hundred amperes flowed. The next step is to measure the necking length of the contact wire. The current can be estimated by comparing the measured result with the approximate curve in Fig. 12.

However, only the approximate curve in case of the new contact wire of GT-SNN100 $\mathrm{mm}^{2}$ and a carbon contact strip has achieved. Characteristics in the case where other types of the contact wire and the contact strip are used and the contact wire is worn will be examined in future.

\section{CONCLUSION}

Understanding of contact-wire breakage phenomenon in the insulated overlap is strongly desired. In this study, we propose a method of estimating the current flowed between the contact wire and the contact strip as the arc in the insulated overlap. The method is to estimate the current by measuring the necking length of the breakage contact wire and comparing it with the approximate curve we obtained in the experiment. The experiment is conducted under the condition that the arc current is set at about 30A to 300A under tension of $9.8 \mathrm{kN}$ or $19.6 \mathrm{kN}$. The experiments revealed the relationship between the arc current and the melted 
marks of the contact wire at the breakage point and the relationship between the arc current and the necking length at the breakage point of the contact wire.

In future, we will conduct heat conduction analysis of the contact wire and compare it with this experiment result. And we want to be able to estimate the current even under other conditions, such as worn contact wire using heat conduction analysis.

\section{REFERENCES}

[1] Ito, K. et al., A study on relation between elongation and arc current when contact wire breaking by an arc. The Institute of Electrical Engineers of Japan, 5(212), pp. 329-330, 2017 (in Japanese).

[2] Denryoku Gijutsu Iinkai., Densyasen [ii], Japan Railway Electrical Engineering Association, pp. 44-47, 2008 (in Japanese).

[3] Hayashiya, H., Gasen-Pantograph Kan Arc Gensyo, Japan Railway Electrical Engineering Association, 2007 (in Japanese).

[4] Fujii, Y. et al., Development of Contact Wire Made of Cu-Ni-Si Alloy. RTRI Report, 17(7), 2003 (in Japanese). 